\title{
The Compound Maintenance Cost Model for Key Equipment with Imperfect Inspection
}

\author{
Bai Yongsheng, Wang Guangyan, Guo Chiming, Tian Xia \\ Department of Management Engineering, Mechanical Engineering College, Shijiazhuang, China
}

\begin{abstract}
Based on the theory of delay time, a compound maintenance strategy, that was block replacement with on-condition tasks, was introduced for keyequipment. The maintenance cost was analyzed, and its mathematical model in the situation of imperfect inspection was established, which took the number and interval of on-condition tasks as variables. On the basis of above research, a numerical example was also given to illustrate the model's application, and the result verified its effectiveness in reducing maintenance cost.
\end{abstract}

KEYWORD: Compound maintenance; Cost Model; Imperfect inspection

\section{INTRODUCTION}

Currently, key equipment is playing a more and more important role in social economics. To ensure the reliability and safety of the key equipment mentioned above, the common situation is, when the key devices are set in run, the preventive maintenances are carried on.

At first, the preventive maintenances used to be on the basis of time, such as periodic replacement. Since the 1980s, the theory of Condition-Based Maintenance (CBM) has been broadly researched and applied in China, and its advantages are also becoming more and more evident. CBM is also known as on-condition tasks, and its implementation is grounded on the thorough understanding of items' failure laws. Owing to CBM, the maintenance is developed to be on the basis of running condition. In practice, there exist the compound maintenance tasks. The compound maintenance means the maintenance mode integrating two or more kinds of maintenance types. For example, the maintenance policy for the transmissions of the locomotive is usually under periodic major repair with some times of preventive minor repair. The researches on such type of maintenance mode are little.

Hence, according to key equipment' failure rules and characteristics, the former maintenance policy, which means scheduled replacement only, has been improved, and on-condition tasks are carried out between the replacement periods to reduce the failure hazard.
In this paper, combined with the typical compound maintenance mode of block replacement with on-condition tasks, the maintenance cost was analyzed for key equipment in the situation of imperfect inspection, and the mathematical models were established. The researches in this paper can assist scientific decision-making to determine the on-condition tasks' interval and its number between two successive replacements.

\section{COMPOUND MAINTENANCE BASED ON DELAY TIME}

\subsection{Delay Time}

In the maintenance research, the items are traditionally considered to have two states: normal and failure. But in practice, failures usually do not occur instantaneously, and they actually develop over a period of time. In 1973, Christer put forward the concept of "delay time", which considers items have three states: normal, potential failure and functional failure. If evidence can be found that this failure process is under way, it may be possible to take relevant action to reduce or avoid the consequences caused by functional failure.

According to the items' failure rules and characteristics, the maintenance policy of scheduled replacement singly is improved, and on-condition tasks are carried out in the replacement period to reduce the failure hazard. 


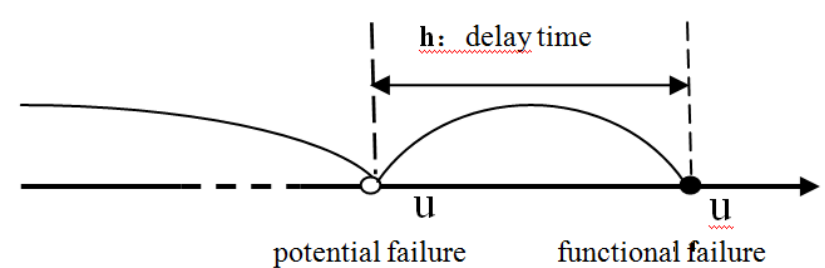

Fig. 1 the concept of delay time

\subsection{Compound Preventive Maintenance Strategy with Imperfect Inspection}

In practical maintenance, to make the best use of component life, reduce failure rate, and save maintenance cost, the compound maintenance type that integrates major and minor repair is usually adopted. The optimization of this kind of maintenance tasks should be further studied and explored.

The maintenance process of compound maintenance is usually as follows: the major maintenance is carried out with pre-set interval, while there are several times of minor maintenance equally between the intervals. Because the major and minor maintenances are usually periodic replacement and on-condition task, the maintenance type of block replacement with on-condition tasks is illustrated to study the compound maintenance.

The detailed process is as follows: the component is preventively replaced with the interval of $T r$, and between replacements the on-condition tasks are implemented with the interval of $\operatorname{Tr} / N$, which means there are $(N-1)$ times of inspections before the replacement(see figure 2). When carrying on inspections, if a potential failure is identified, preventive maintenance should be adopted; if not, the component will continue to work until either a failure occurs or the next check. During the replacement period, if a functional failure occurs, the item should be repaired.

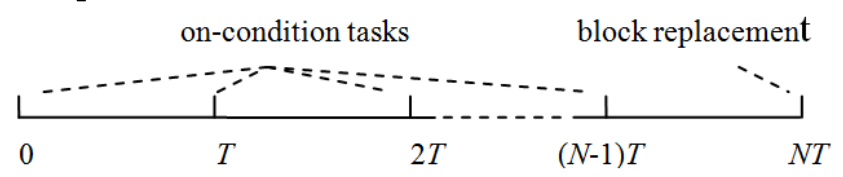

Fig.2 Block replacement with on-condition tasks

In practice, the skill base which may affect the inspection quality may be lacking for the first few inspections, so the assumption of perfect inspection may be suspect in the finite time horizon modeling. In this paper, we assume the inspection is imperfect with a detection rate $r$ and seek the optimum inspection interval.For the sake of simplicity, assuming the conventional case where the potential failure is non-measurable, the inspection intervals are constant, so the decision variable in the model is the inspection interval and replacement interval. It is obvious that these assumptions can be relaxed by considering the measurable potential failure and first inspection interval in the model.

\section{MODELING MAINTENANCE COST FOR COMPOUND MAINTENANCE WITH IMPERFECT INSPECTION}

For key equipment, as viewed from infinite time horizon, the items are under block replacement policy, so the aim is to optimize the replacement period minimizing average cost per unit time.

\subsection{Notation and Assumption}

- The key equipment is considered as a single component with single failure mode.

- Either failure renewal or inspection renewal makes the key equipment as good as new.

- The average time of inspection is far shorter than that of inspection interval, and can be ignored.

- $\quad U$ : The time when potential failures arise, and its p.d.f and c.d.f are denoted by $g(u)$ and $G(u)$, respectively.

- $H$ : The delay time from potential failure to functional failure, and its p.d.f and c.d.f are denoted by $f(h)$ and $F(h)$, respectively.

- $\quad r$ : The detection rate of inspection, for it is imperfect.

The decision variables are as follows:

- $T$ : Functional check interval.

- $\quad N$ : The number of inspection intervals contained in a block replacement period.

The probabilities of events are denoted as follows:

- $P_{n}(N T)$ : The probability of no renewal event over block replacement period $N T$.

- $\quad P_{m}(i T)$ : The probability of inspection renewal at $i T$.

- $\quad p_{b}(x)$ : The p.d.f of failure renewal at $x$.

The costs of events are denoted as follows:

- $C_{\mathrm{p}}, C_{i}, C_{f}, C_{c}, C_{d}$ : The average cost of a preventive maintenance, inspection, failure repair, failure compensation and block replacement.

- $C(t)$ : The expected cost during $[0, t]$.

\subsection{Maintenance Cost Analysis}

According to the renewal reward theorem, the mean maintenance cost per unit time can be expressed as: 


$$
\operatorname{Min}\left[\lim \frac{C(t)}{t}\right]=\operatorname{Min}\left[\frac{C(N T)+C_{d}}{N T}\right]
$$

Where $C(N T)$ denote the expected cost during [0, $N T$ ] except the cost of block replacement.

During every block replacement period $[0, N T]$, the items are under imperfect age inspection policy for finite time horizon. Therefore, the cost $C(N T)$ is made up of the following three events, one of which must occur over a finite time NT:

(1): Neither inspection renewal nor failure renewal occurs during block replacement period, that is, there is no renewal event over NT. The cost can be expressed as: $(N-1) \cdot C_{i}+C_{d}$,

(2): Renewal events occur, and the first renewal is an inspection renewal at $i T$, the $i t h$ inspection. The cost can be expressed as: $i \cdot C_{i}+C_{p}+C(N T-i T)$.

(3): Renewal events occur, and the first renewal is a failure renewal at time $x((i-1) T<x<i T)$. In view of the compensation, the cost can be expressed as: $(i-1) C_{i}+C_{f}+C_{c}+C(N T-x)$

\subsection{Maintenance Cost Modelling}

The probabilities of events analyzed above can be deduced as follows:

(1) The event that there is no renewal event over NT may be resulted from the following two cases:

Case 1: there is no potential failure occurring before $N T$, i.e. case $1=U \geq N T$;

Case 2: a potential failure occurs at $\mathrm{u}$ between two successive checks, say $((j-1) T, j T)$, and this potential failure is not identified by the coming $(N$ $j+1)$ checks and there is no functional failure occurring during $(N T$ - $u)$, i.e. case $2=(j-1) T<U<j T$ $\cap U+H>N T \cap$ not being identified before NT.

Therefore, we have the probability of no renewal event occurring before $N T$

$$
P_{n}(N T)=1-\int_{0}^{N T} g(u) d u+\sum_{j=1}^{N} \int_{t_{j-1}}^{t_{j}}(1-r)^{N-j} g(u)[1-F(N T-u)] d u
$$

(2) To derive the probability of an inspection renewal at time $i T$, we note that in the situation of imperfect inspection, the condition for a defect occurring in $(u, u+d u)\left(t_{j-1}<u<t_{j}\right)$ and being identified at ith inspection is a combination of the following three events:

- The defect was not identified before $(i-1) T$ by $(i-j)$ inspections.

- The defect was identified at the ith inspection.

- The delay time of the defect must be longer than $i T-u$.

The probability of this event is:

$$
g(u) d u(1-r)^{i-j} r[1-F(i T-u)] \text {. }
$$

Integrating all possible u between $((N-1) T, N T)$, we have the probability of a defect being identified at inspection $i T$ as

$$
P_{m}(i \cdot T)=\sum_{j=1}^{i}(1-r)^{i-j} r \int_{(j-1) T}^{j T} g(u)[1-F(i T-u)] d u
$$

(3) To derive the p.d.f of a failure renewal at time $x$, we assume that a defect arises at $(u, u+d u)$ $((i-1) T<u<i T)$, for it is to become a failure in $(x$, $x+d x)$, the delay time $h$ must satisfies $x-u<h<x+d x-u$. So the probability density is:

$$
p_{b}(x)=\sum_{j=1}^{i-1}(1-r)^{i-j} \int_{(j-1) T}^{j T} g(u) f(x-u) d u+\int_{(i-1) T}^{x} g(u) f(x-u) d u
$$

Considering all the events that can take place at any time over $N T$, we have the cost function as:

$$
\begin{aligned}
& \mathrm{C}(\mathrm{NT})=\left[(\mathrm{N}-1) \bullet C_{i}\right] \bullet P_{n}(\mathrm{NT}) \\
& +\sum_{i=1}^{N-1}\left[i \cdot \mathrm{C}_{\mathrm{i}}+C_{P}+C(N T-i T)\right] \bullet P_{m}(i \cdot T) \\
& +\sum_{i=1}^{N} \int_{(i-1) T}^{i T}\left[(i-1 \cdot) \mathrm{C}_{\mathrm{i}}+C_{f}+C_{c}+C(N T-x)\right] \bullet p_{b}(x) d x
\end{aligned}
$$

By the equations (2)-- (5), we can get the function for average cost per unit time: $\frac{C(N T)+C_{d}}{N T}$, then the optimal interval $\mathrm{T}$ in equation (1) can be obtained.

\section{A NUMERICAL EXAMPLE}

\subsection{Example Analysis}

The maintenance policy of an item in the motorcycle is that, there are four times of on-condition tasks in a block replacement period. It's investigated that $C_{p}=\$ 5000, C_{i}=\$ 500, C_{f}=\$ 15000, C_{c}=\$ 5000$, $C_{d}=\$ 3000$ and $r=0.8$. In this paper, $U$ and $H$ are assumed to follow weibull and exponential distribution, respectively; and the values of relevant parameters are $\alpha_{u}=2.5, \beta_{u}=16$ days and $\lambda_{h}=22$ days. We seek the interval of on-condition tasks to minimize expected cost.

Evidently, the present maintenance policy is under block replacement with four times of oncondition tasks. Firstly, we should find out with how many times of on-condition tasks the cost could be minimized; then on its basis, the inspection interval should be optimized in order to minimize the average cost per unit time.

\subsection{Model-assisted Decision}

By calculating the example with the model above, a curve can be obtained about the average cost per unit time and inspection interval with MatLab 7.1 (see Figure 3). Therefore we can conclude that: 
The results of the model indicate that the value of minimum cost $\operatorname{Min}\left[\frac{C(N T)+C_{d}}{N T}\right]$ is variable with the increase of $N$. In this case, when $N=6$, $\operatorname{Min}\left[\frac{C(N T)+C_{d}}{N T}\right]$ is minimum, which equals to $\$ 166.22 /$ day.

When $N=1$, it represents that there is only block replacement without on-condition tasks, the minimum average cost per unit time are distinctly higher. $\operatorname{Min}\left[\frac{C(T)+C_{d}}{T}\right]$ reached the least $\$ 203.45 /$ day when $T=57$. The conclusion also shows the effectiveness of on-condition tasks.

If the policy is adjusted to contain four times of on-condition tasks in every period of block replacement, i.e. $N=6$, the optimal interval of oncondition tasks $T^{*}$ is 24 days.

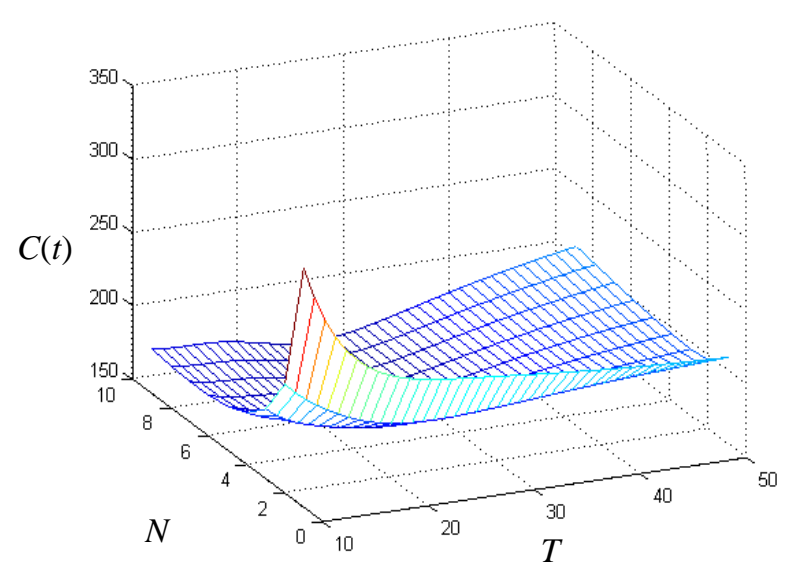

Fig 3 The three-dimensional diagram of cost optimization

Thus it can be seen that, if on-condition tasks are artificially carried out excessively or insufficiently, the cost would not be reduced, on the contrary, it might be increased sharply, which might even disturb its normal function or decrease its reliability.

\section{CONCLUSIONS}

Aiming to the requirements of preventive warranty for key devices, the compound preventive maintenance policy is introduced to optimize the number and intervals of on-condition tasks.. Taking the block replacement with on-condition tasks as example, the mathematical model for maintenance cost is established, and the effectiveness is validated by a case study. Actually, the model is established only from the aspect of cost; if the failure consequences are evaluated by other factors, such as availability, risk and so on, the corresponding models can also be established in the similar way. The researches on preventive maintenance modeling could provide references for maintenance decision; furthermore, they are of great significance for improving decision scientificity and practical application.

\section{REFERENCES}

[1] Jia Xisheng. The Decision Models for Reliability Centered Maintenance . National Defense Industry Press, 2007.

[2] Renyan Jiang, D.N.Prabhakar Murthy. Maintenance: decision models for management . Science Press, 2008: 127-133.

[3] Gan Maozhi, Kang Jianshe and Gao Qi, Military Equipment Maintenance Engineering, Second Edition. Beijing: National Defense Industry Press, 2005.

[4] Oguzhan Yilmaz, Nabil Gindy, Jian Gao. "A repair and overhaul methodology for aeroengine components". Robotics and Computer-Integrated Manufacturing, 2010, 26:190-201.

[5] Yongsheng Bai, Xisheng Jia, Zhonghua Cheng. "A Cost Model of Block Replacement with Functional Checks". Proceedings of the First International Conference on Maintenance Engineering, Chengdu, China, 2006. Beijing: Science Press.

[6] Renyan Jiang and D. N. Prabhakar Murthy, Maintenance Decision Models for Management. Beijing: Science Press, pp.127-133, 2008.

[7] Zhao Jianhua, Zhao Jianmin and Zhao Liqin, "An optimization of joint preventive maintenance for a multicomponent system". Mathematics in Practice and Theory, vol.35, no.6, pp.182-188, 2005. 